\title{
Spectroscopy made easy: A new tool for fitting observations with synthetic spectra
}

\author{
J.A. Valenti ${ }^{1}$ and N. Piskunov ${ }^{2}$ \\ 1 JILA, University of Colorado, Boulder, Colorado 80309-0440, U.S.A. \\ 2 Astronomical Observatory of Uppsala University, Box 515, S-751 20 Uppsala, Sweden
}

Received November 22; accepted January 19, 1996

\begin{abstract}
We describe a new software package that may be used to determine stellar and atomic parameters by matching observed spectra with synthetic spectra generated from parameterized atmospheres. A nonlinear least squares algorithm is used to solve for any subset of allowed parameters, which include atomic data (log $g f$ and van der Waals damping constants), model atmosphere specifications $\left(T_{\text {eff }}, \log g\right)$, elemental abundances, and radial, turbulent, and rotational velocities. LTE synthesis software handles discontiguous spectral intervals and complex atomic blends. As a demonstration, we fit 26 Fe I lines in the NSO Solar Atlas (Kurucz et al. 1984), determining various solar and atomic parameters.
\end{abstract}

Key words: radiative transfer - methods: numerical — techniques: spectroscopic — stars: fundamental parameters — sun: fundamental parameters — atomic data

\section{Introduction}

Traditional stellar spectroscopy may be divided into four steps: spectroscopic observation, data reduction, measurement of stellar parameters (e.g. rotational velocities, effective temperature, abundances), and astrophysical interpretation. Large telescopes, high resolution spectrographs, and digital detectors have made it possible to routinely obtain spectra at a resolution of 200000 with noise below $0.3 \%$ for stars down to 8 th magnitude. Reduction software has kept pace with improvements in instrumentation, but we have yet to take full advantage of modern computers in the measurement phase of the analysis. There is no reason to discard the rich information content in modern spectra, simply because traditional analyses only use equivalent widths. By matching synthetic spectra to the observed spectrum, we can minimize errors due to blends and continuum placement. In addition, modern computers make it possible to search parameter space for the model which best matches the observations. This approach allows one to characterize errors in each parameter and decide whether it is better to fix a parameter to an externally determined value or to solve for the parameter using the observed spectrum as a constraint.

In this paper, we describe a new software package (Spectroscopy Made Easy, SME) which allows one to find the best fit to an observed spectrum for a given set of

Send offprint requests to: J.A. Valenti assumptions. Remaining discrepancies may then be interpreted as inaccuracies in atomic line parameters and/or inadequacies in the adopted model atmosphere (e.g. granulation is ignored). SME can be used to determine atomic parameters (log $g f$ and van der Waals damping) for individual transitions and/or global stellar parameters ( $V_{\mathrm{rad}}$, $V \sin i, T_{\text {eff }}, \log g$, abundances, $V_{\text {micro }}$, and $\left.V_{\text {macro }}\right)$. SME may be divided conceptually into three main components: a spectrum synthesis library written in $\mathrm{C}++$, parameter optimization code written in IDL and intended for batch execution, and a widget-based user interface which is also written in IDL. Analysis tools with related goals, but somewhat different approaches are being developed by Hill (Hill et al. 1996) and Cowley (Cowley 1996). Below, we describe how SME works and illustrate its use with an analysis of the solar spectrum.

\section{Radiative transfer}

Spectral synthesis is the most computationally intensive part of SME, so we use a very fast algorithm adapted from the program SYNTH (Piskunov 1992). Currently in SME, we assume: local thermodynamical equilibrium (LTE), plane parallel geometry, no bulk flows, no molecular line opacity, and negligible magnetic field. SME handles depth dependent microturbulence that is (unlike SYNTH) the same for all lines. 
The spectral synthesis code is implemented as a $\mathrm{C}++$ library, which is dynamically linked with IDL at execution time. This allows us to combine the speed of $\mathrm{C}++$ with the high level functionality and user interface of IDL. The $\mathrm{C}++$ library contains modules for computing ionization balance, continuous opacities, emergent intensities, and contribution functions, as well as I/O modules for communicating with IDL.

\subsection{Data input}

Before the $\mathrm{C}++$ library can calculate an intensity spectrum, four types of information must be specified: a model atmosphere, elemental abundances, atomic line data, and a set of wavelength intervals. Because of the perturbative nature of the nonlinear least squares algorithm described below, SME can often make use of certain results from preceding calculations. Accordingly, each type of data required by the $\mathrm{C}++$ library can be modified independently from IDL, allowing a partial respecification of a radiative transfer request. The radiative transfer code checks that all required data have been supplied and then calculates any quantities that have been invalidated by new request specifications. For example, changing the atomic parameters for one line has no effect on ionization equilibrium and affects the intensity spectra in only one wavelength interval. Adding a line from a previously unrepresented element, however, will force a recalculation of the ionization balance and all subsequent steps.

\subsection{Ionization equilibrium and continuous opacity}

Ionization equilibrium is determined by solving the Saha equation:

$$
\frac{N_{i}}{N_{i-1}}=\frac{(2 \pi k T)^{3 / 2}}{h^{3} N_{\mathrm{e}}} \cdot \frac{2 U_{i}}{U_{i-1}} \exp \left(-\frac{\Delta E}{k T}\right),
$$

which gives the ratio of two consecutive ionization states as a function of electron density and temperature. For the partition functions, $U$, we use polynomial approximations which describe the first six ionization stages of the first 99 elements in the periodic table (Kurucz 1979).

Ionization fractions are computed on a finer depth grid than that of the original model atmosphere because ionization can change more rapidly than other quantities. The number of subdivisions is determined at each depth to ensure that the the fractional change of each ionization state is less than $10 \%$. Normally, only a few depth intervals need to be divided into a maximum of 20 subintervals each in order to satisfy this condition. The temperature and electron density in each subinterval is determined by parabolic interpolation in the original grid. Ionization balance is determined for hydrogen, helium, and every other element present in the line list. Neutral hydrogen and helium populations are used later for computing collisional line broadening.
Continuous absorption coefficients (in $\mathrm{cm}^{2} \mathrm{~g}^{-1}$ ) are computed at the endpoints of each wavelength interval, using the ionization equilibrium depth grid. Continuous opacity routines were adapted from the ATLAS 9 spectrum synthesis code (Kurucz 1993).

\subsection{Solution of radiative transfer equation}

Specific intensities are determined by solving the integral form of the radiative transfer equation:

$$
I_{\lambda}=\int_{0}^{\infty} \mathrm{e}^{-\tau / \mu} S_{\lambda}(\tau) \mathrm{d} \tau
$$

where $\tau$ is the optical depth and $S_{\lambda}(\tau)$ is the source function (i.e. the Planck function, $B_{\lambda}$, given our assumption of LTE). Equation (2) is solved using Gaussian quadrature:

$$
I_{\lambda}=\sum_{i=1, N} B_{\lambda}\left(\tau_{i}\right) \cdot w_{i}
$$

where $\tau_{i}$ and $w_{i}$ are the quadrature nodes and weights (see Table 1) for a basis set of Hermite polynomials. We find that 10th order quadrature gives fractional integration errors less than $210^{-5}$ for realistic model atmospheres.

Table 1. Nodes and weights of 10th order quadrature formula used for solving the radiative transfer equation

\begin{tabular}{cc}
\hline \multicolumn{1}{c}{$\tau_{i}$} & $w_{i}$ \\
\hline 0.137793470540 & $3.08441115765 \mathrm{e}-01$ \\
0.729454549503 & $4.01119929155 \mathrm{e}-01$ \\
1.808342901740 & $2.18068287612 \mathrm{e}-01$ \\
3.401433697855 & $6.20874560987 \mathrm{e}-02$ \\
5.552496140064 & $9.50151697518 \mathrm{e}-03$ \\
8.330152746764 & $7.53008388588 \mathrm{e}-04$ \\
11.843785837900 & $2.82592334960 \mathrm{e}-05$ \\
16.279257831378 & $4.24931398496 \mathrm{e}-07$ \\
21.996585811981 & $1.83956482398 \mathrm{e}-09$ \\
29.920697012274 & $9.91182721961 \mathrm{e}-13$ \\
\hline
\end{tabular}

The model atmosphere is tabulated either as a function of optical depth $\left(\tau_{\text {ref }}\right)$ at a reference wavelength (typically at $5000 \AA)$ or mass column density $(\rho d x)$. For each wavelength point in the spectrum, $B_{\lambda}$ must be calculated at the optical depths, $\tau_{i}$, used in the quadrature. Conversion between optical depth, $\tau_{\lambda}$, and the model depth parameter is accomplished by solving one of two first order differential equations:

$$
\begin{aligned}
\mu \frac{\mathrm{d} \tau_{\text {ref }}}{\mathrm{d} \tau_{\lambda}} & =\frac{K_{\text {ref }}}{K_{\lambda}+A_{\lambda}}, \\
\mu \frac{\rho d x}{\mathrm{~d} \tau_{\lambda}} & =\frac{1}{K_{\lambda}+A_{\lambda}},
\end{aligned}
$$

where either Eqs. (4) or (5) is used, depending on whether $\tau_{\text {ref }}$ or $\rho d x$ is the independent depth parameter. In these 
equations, $K_{\lambda}$ and $K_{\text {ref }}$ are the continuous opacities (in $\mathrm{cm}^{2} \mathrm{~g}^{-1}$ ) at $\lambda$ and $\lambda_{\text {ref }}$, and $A_{\lambda}$ is the line opacity given by

$$
A_{\lambda}=\frac{1}{\rho} \sum \frac{\pi e^{2}}{m c} g f N \frac{\lambda^{2}}{\sqrt{\pi} c \Delta \lambda_{D}}\left(1-\mathrm{e}^{-\frac{h c}{\lambda k T}}\right) H(a, v)
$$

where $g, f$, and $N$ are the statistical weight (degeneracy) of the lower level, the oscillator strength, and the number density of the atoms in the lower level. The summation is over all lines contributing at given wavelength. The Voigt function,

$$
H(a, v)=\frac{a}{\pi} \int_{-\infty}^{\infty} \frac{\exp \left(-y^{2}\right)}{(v-y)^{2}+a^{2}} \mathrm{~d} y
$$

is evaluated as a function of depth, using the Humlíček approximation (Humlíček 1982).

A 6th order Runge-Kutta technique with appropriate boundary conditions is used to integrate Eqs. (4) or (5) and find the value of the independent depth parameter at each of the quadrature nodes. Integration is done on the depth grid constructed in the ionization equilibrium calculation (Sect. 2.2). This approach has two major advantages over direct integration of Eq. (2). First, $\tau_{\text {ref }}$ is a smooth, monotonic function of $\tau_{\lambda}$ that is well sampled on the finer depth grid. Second, the integration progresses downwards from the surface, so it can be truncated when $\tau_{\lambda} \gg 1$.

For each Runge-Kutta step, we interpolate the main atmospheric variables: temperature, number densities of electrons and atoms, ionization fractions for contributing lines, and microturbulence. We calculate Voigt profiles, including the effects of radiative damping, the quadratic Stark effect, and van der Waals broadening. Damping constants $\left(\Gamma_{\mathrm{rad}}, \Gamma_{4}, \Gamma_{6}\right)$ that are not provided with the line list are approximated using formulas from Unsöld (1955), Cowley (1971), or Griem (1968). Stark broadening in hydrogen and helium must be treated as a special case. For the Balmer series of Hydrogen, we use the approximation of Vidal et al. (1971), and for 20 optical lines of He I, we use tables of Stark broadening and central wavelength shifts calculated by Dimitrijević (1971).

Synthetic spectra are computed on an adaptive wavelength grid to produce uniform precision when interpolating the spectrum. We calculate the specific intensity at the endpoints of each wavelength interval and at each line center. We then insert new wavelength points midway between existing points until the accuracy of the interpolated profile is better than a selectable threshold (see Vincent et al. 1993 for more details). This algorithm results in more wavelength points where the spectrum has higher curvature.

The ultimate precision of the synthetic spectrum at an arbitrary wavelength is controlled by two factors: the precision of the radiative transfer solution at each point in the irregularly spaced wavelength grid, and the interpolation accuracy between these grid points. We can achieve a fractional precision of $10^{-4}$, which is more than adequate in most situations, given current uncertainties in model atmospheres and line formation mechanisms.

\section{Fitting the observations}

\subsection{Atomic parameters}

SME requires line data for all atomic transitions of interest. Mandatory data are element name, ionization state, wavelength, excitation energy of the initial state, and $\log g f$. Default approximations are used to estimate the radiative, Stark, and van der Waals damping constants, if they are not explicitly specified. SME initially reads atomic data from one or more text files in the format returned by a "stellar" request to the Vienna Atomic Line Database (VALD, Piskunov et al. 1995) electronic mail server. VALD critically selects atomic line data for those transitions that contribute significantly to the spectral synthesis. Once loaded into SME, atomic data may be saved in an internal format for later use.

SME can be used to solve for $\log g f$ values and/or van der Waals damping constants. Parameters may be varied individually on a line by line basis or globally via a correction to all van der Waals damping constants. Global enhancement of the van der Waals damping predicted by Unsöld (1955) is common practice, for example when modeling neutral iron lines (Holweger et al. 1991). Using SME, atomic parameters may be adjusted to best match an observed spectrum of some star (the Sun, for example). The new "astrophysical" atomic parameters are often an improvement over theoretical values or low quality laboratory data, and can be used in subsequent analyses of other stars.

\subsection{Model parameters}

In order to synthesize a spectrum, SME requires a model atmosphere. The required atmospheric data are temperature, electron number density, atomic number density, and mass density. These data are specified on a depth grid parameterized either by integrated mass column or by continuum optical depth at a reference wavelength (5000 $\AA$, for example). SME could easily be modified to calculate (rather than read) densities, as long as radiation pressure is negligible.

Model atmospheres are often tabulated on a grid of $\left\{T_{\text {eff }}, \log g,[\mathrm{Fe} / \mathrm{H}]\right\}$. SME uses bicubic spline interpolation of the logarithm of each atmospheric variable to generate an atmosphere for any desired $\left\{T_{\text {eff }}, \log g,[\mathrm{Fe} / \mathrm{H}]\right\}$ contained within the limits of the grid. The interpolated model is constructed on the mass column (or continuum optical depth) scale of the closest model in the grid. Grid interpolation makes it easier to generate an atmospheric 
model for a specific $\left\{T_{\text {eff }}, \log g,[\mathrm{Fe} / \mathrm{H}]\right\}$ or to solve for the $\left\{T_{\text {eff }}, \log g,[\mathrm{Fe} / \mathrm{H}]\right\}$ that give the best agreement with an observed spectrum.

\subsection{Disk integration of specific intensity}

Specific intensities must be integrated over the stellar surface to produce a flux spectrum. Techniques for doing this vary in both speed and accuracy. For nonrotating stars, specific intensity depends only on $\mu$, making onedimensional Gaussian quadrature accurate and efficient. For rotating stars, annular regions of constant $\mu$ span a range of rotational velocities, and a two-dimensional surface integral is formally required to compute the flux. To increase the speed of this calculation, one may first calculate the flux profile ignoring rotation and then convolve the flux profile with a broadening kernel designed to mimic the effects of rotation. This approximation is inadequate for precise spectroscopy of stars rotating at less than about $15 \mathrm{~km} \mathrm{~s}^{-1}$ (Gray 1992).

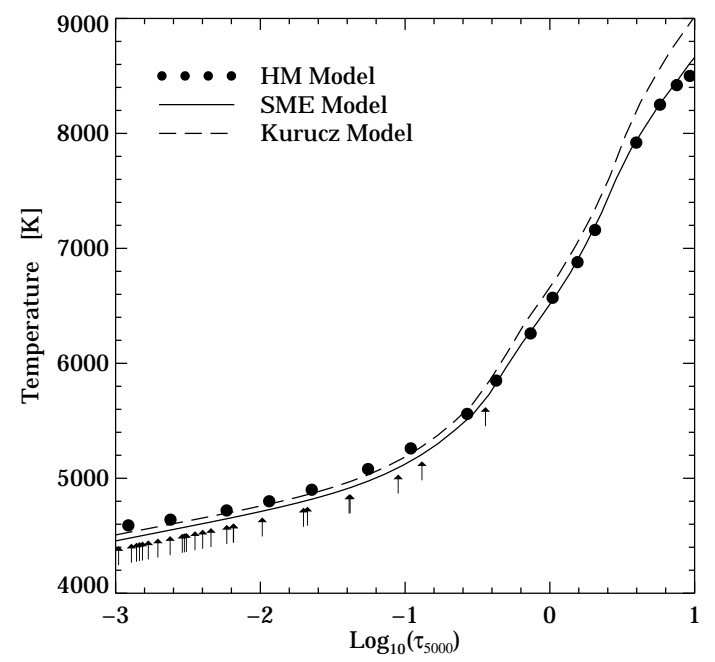

Fig. 1. Comparison of the temperature distributions for the HM, SME, and Kurucz solar models. Arrows indicate the depths of formation for the cores of each of the $26 \mathrm{Fe}$ I lines in our analysis

In SME we employ a recently developed disk integration technique in which the star is divided into annular regions, each characterized by a single intensity spectrum. The effects of solid-body rotation are handled by convolving each intensity spectrum with an analytic kernel that properly weights the velocity shifts in the corresponding annulus. In this manner, calculation of the flux spectrum is reduced to a one-dimensional sum of convolutions, resulting in good speed and excellent accuracy (Valenti \& Anderson 1995). By default SME uses seven $\mu$ angles, which gives an integration accuracy better than $0.1 \%$ in most circumstances. The number of $\mu$ angles can be changed to vary the speed and accuracy of calculation. The effects of isotropic Gaussian macroturbulence are treated by a single convolution of the rotationally broadened flux profile. When radial and tangential macroturbulent velocities are unequal, each annulus in the disk integration has its own macroturbulent broadening kernel.

\subsection{Matching the observations}

SME simplifies the determination of atomic and stellar parameters by using an observed spectrum as a model constraint. When empirically matching an observed spectrum, the behavior of the spectrograph and detector must be included in the model. An instrumental profile is specified in tabular form or as an analytic function (a Gaussian, for example). The synthetic spectrum is spline interpolated onto a uniform wavelength grid with points spaced finely enough to preserve all information contained in both the model spectrum and the instrumental profile. The instrumental profile is interpolated onto (or generated on) the same wavelength grid and convolved with the synthetic spectrum.

Each segment of synthetic spectrum is independently shifted in wavelength and linearly scaled in amplitude until $\chi^{2}$ is minimized. For each trial wavelength shift, scaling and slope adjustment of the model spectrum are found directly by linear least squares matching of observed "continuum" points. If necessary, the synthetic spectrum is integrated over each observed pixel to mimic wavelength binning by the detector. Continuum points are specified explicitly by the user or determined automatically by SME, based on how close the points lie to the maximum flux in the spectral segment. The presence of spectral lines in the "continuum" fitting regions only affects continuum placement if the depth of model lines is wrong.

To find the optimum wavelength offset, we calculate $\chi^{2}$ for a sequence of wavelength shifts (and corresponding continuum adjustments). A parabola is fit to $\chi^{2}$ as a function of wavelength shift. The parameters at the minimum of the parabola are then used to match the synthetic spectrum to the observations. The wavelength adjustments measure and correct errors in radial velocity, transition wavelengths of the dominant lines, and zero point errors in the observed wavelength scale. The continuum adjustments mimic wavelength variations in spectrograph throughput and correct for errors in observed continuum placement and/or model continuous opacities.

\subsection{Parameter optimization}

As indicated in Sects. 3.1 and 3.2, SME can solve for various atomic and/or model parameters, using an observed spectrum as a constraint. Free parameters are optimized simultaneously, using a $\chi^{2}$ minimization algorithm described by Marquardt (Marquardt 1963; Press et al. 1986). Sequential optimization of different sets of parameters may be done by running SME once for each parameter set. 


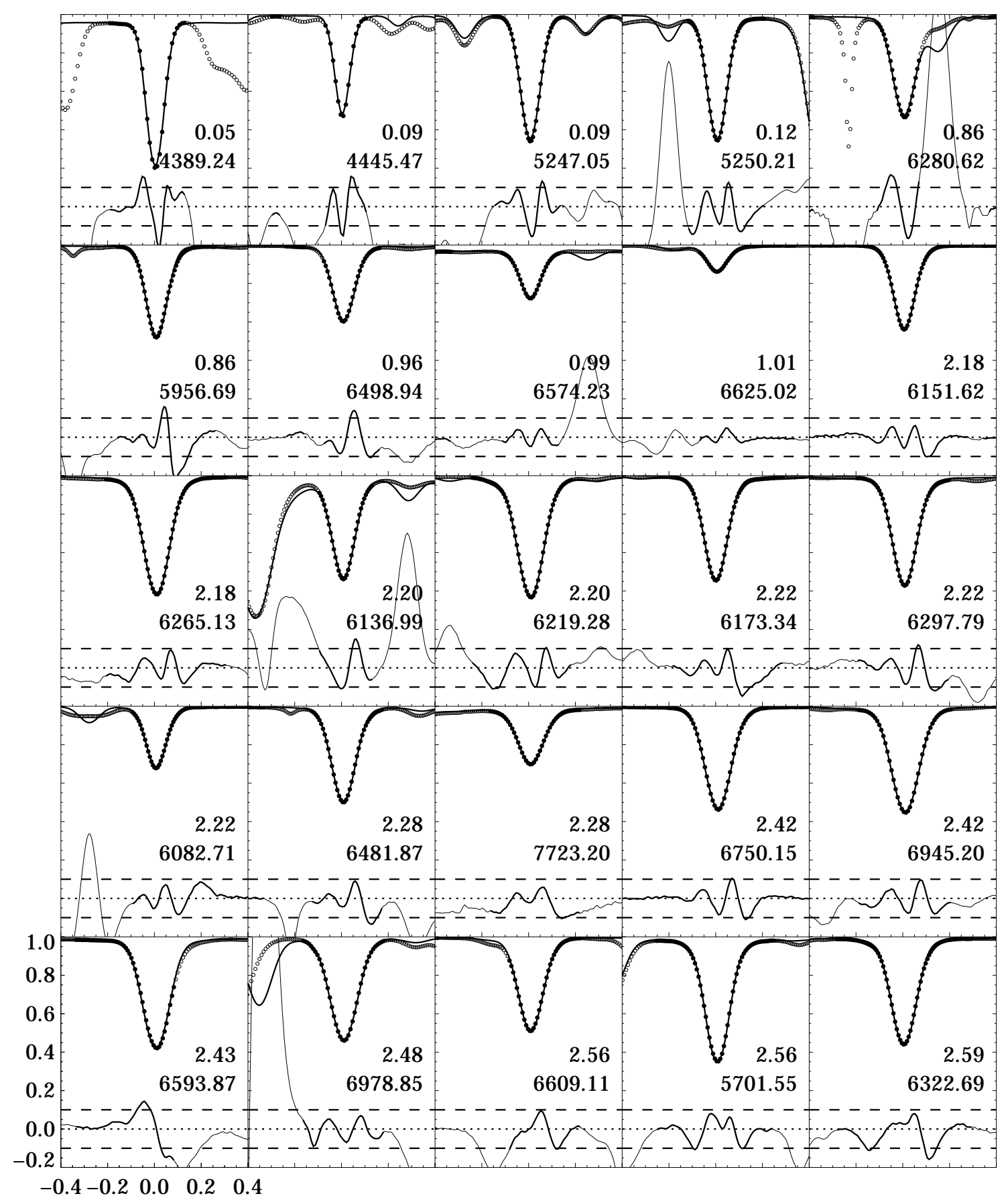

Fig. 2. A comparison of $25 \mathrm{Fe}$ I observed and model line profiles after adjusting solar parameters and individual oscillator strengths. The top of each panel shows the synthetic spectrum (solid line) and the observations (filled circles for points fit by SME; open circles for points that were ignored). The bottom shows residuals multiplied by 10, where the thicker line corresponds to the solid circles above. Horizontal dashed lines indicate $\pm 1 \%$ error levels. Panels are sorted by (and labelled with) excitation potential. Central wavelengths (in $\AA$ ) are also given. Wavelength offsets (in $\AA$ ) and residual intensity for all lines are indicated in the bottom left hand panel 
The Marquardt algorithm mixes a traditional gradient search with approximate parabolic minimization of the $\chi^{2}$ surface near the minimum. Approximate second partial derivatives in the curvature matrix are constructed from first partial derivatives, which in turn are found by recomputing synthetic spectra at small offsets in each free parameter. For each set of partial derivatives, three to five model calculations are required to choose a good mix between a gradient search and parabolic minimization. The new parameters are used to recalculate partial derivatives and the process repeats until $\chi^{2}$ changes by less than some fractional amount, typically $0.2 \%$.

Convergence to a fractional limit of $0.2 \%$ typically requires only two to five major iterations (for which new partial derivatives are recalculated). As a rule of thumb, we find that model convergence requires about $3.5 N+13$ model spectrum calculations. The inverse of the final curvature matrix is the covariance matrix, which contains formal errors for each parameter and degeneracies between each pair of parameters.

\section{Solar example}

To illustrate the capabilities of SME, we solved for certain solar and atomic parameters by matching synthetic spectra to a flux spectrum of the Sun (Kurucz et al. 1984). We concentrated on a set of 26 Fe I lines with precisely measured laboratory oscillator strengths (Blackwell et al. 1995, BLS and references therein). The equivalent widths of these lines were used by BLS to determine the abundance of iron in the Sun. For demonstration purposes, we decided to repeat their analysis, using SME to match line profiles. The results are shown in Figs. 2 and 3. After completing our tests, we learned that Holweger et al. (1995, HKB) strongly disagree with various interpretations in BLS. It is not our goal here to try and resolve this debate, but we do make a few specific comments below, based on our tests with SME.

\subsection{Observational and atomic data}

We used a continuum normalized spectrum of the entire solar surface (Kurucz et al. 1984) as input data for SME. The noise in this FTS spectrum is 0.1 to $0.2 \%$ with a spectral resolving power of $5.210^{5}$. We ignored instrumental broadening, which is negligible since the instrumental profile is one-tenth as wide as the spectral lines. Blends in the wings of the $26 \mathrm{Fe}$ I lines were identified by comparing an initial model spectrum with observations. Blends which were poorly modeled due to inaccurate or missing atomic data were marked and ignored in subsequent analyses.

Atomic data were obtained from VALD for $5 \AA$ wide spectral intervals containing the 26 Fe I lines studied by BLS. We used VALD "stellar" requests to obtain the best available atomic data for 539 lines in these spectral in-
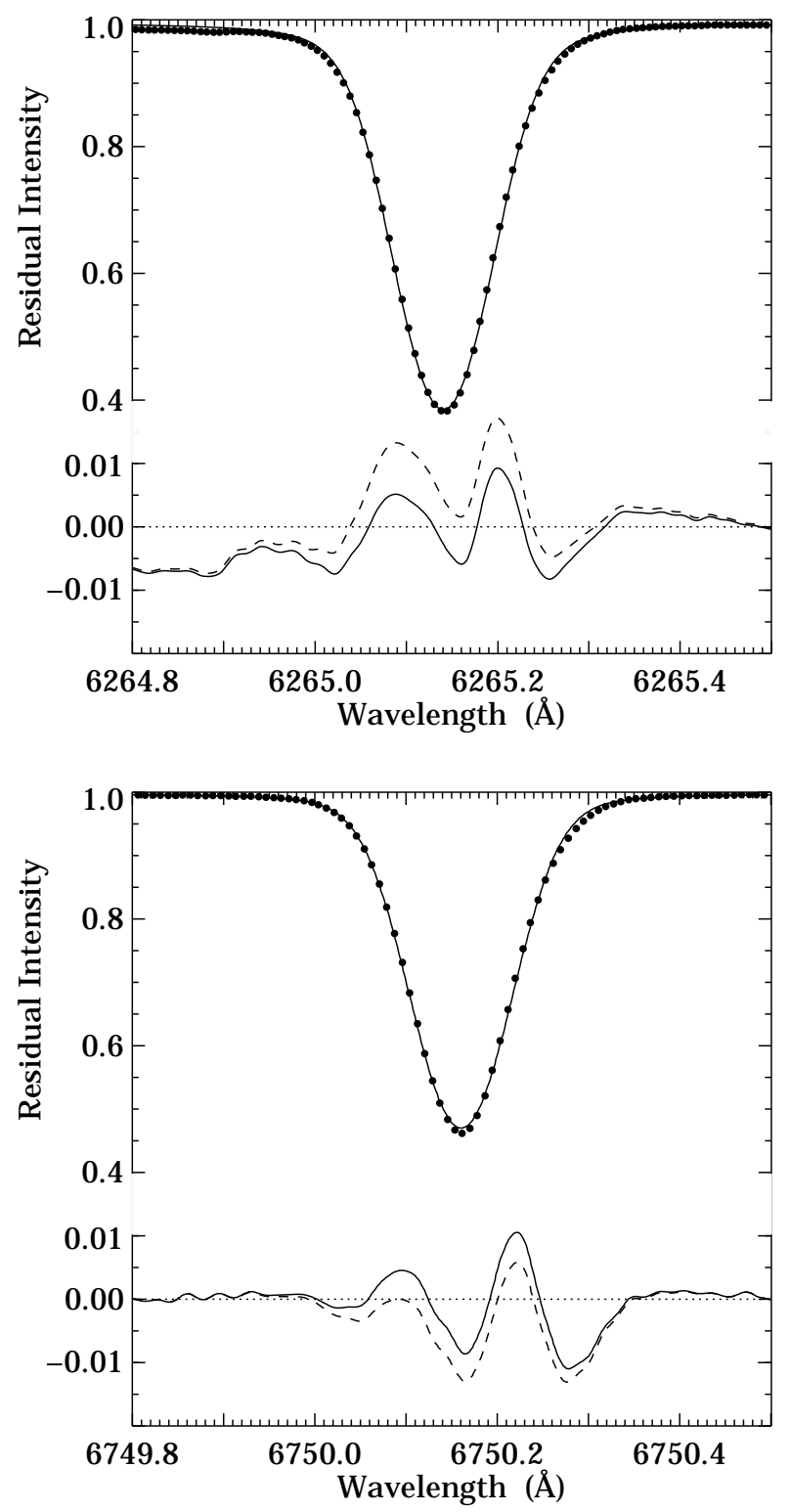

Fig. 3. The same comparison as in Fig. 2 for two of the lines. The residuals are shown for laboratory $\log g f$ (dashed line) and astrophysical $\log g f$ determined by SME (solid line)

tervals with expected fractional depths greater than $0.2 \%$ in the Sun. For the $26 \mathrm{Fe}$ I lines used in the abundance analysis, we adopted Oxford $\log (g f)$ values as tabulated in BLS, except that $\log (g f)=-5.040$ (not -5.004$)$ for the line at $6574.24 \AA$. Damping constants from Kurucz CDROM 18 were initially provided by VALD, though the van der Waals damping constants were subsequently increased by SME, as described below.

\subsection{Determining a solar model}

SME makes it relatively easy to solve for any combination of stellar and atomic parameters, but it doesn't simplify 
the decision of which parameters to vary and which to hold constant. The constraints provided by the observed spectrum lead to a certain accuracy for each derived parameter. If a particular parameter is known more accurately from previous work, then the parameter should be set to the known value. Since accuracy (unlike precision) can be difficult to assess, the decision about which parameters to fix is often subjective.

Table 2. Derived parameters of the solar models

\begin{tabular}{ccccccc}
\hline $\begin{array}{c}T_{\text {eff }} \\
(\mathrm{K})\end{array}$ & $\mathrm{Fe} / \mathrm{H}$ & $\begin{array}{c}V_{\text {micro }} \\
(\mathrm{km} / \mathrm{s})\end{array}$ & $\begin{array}{c}V_{\text {macro }} \\
(\mathrm{km} / \mathrm{s})\end{array}$ & $\begin{array}{c}V \sin i \\
(\mathrm{~km} / \mathrm{s})\end{array}$ & $\Delta \Gamma_{6}$ & $\begin{array}{c}\mathrm{RMS} \\
(\%)\end{array}$ \\
\hline 5734 & -4.50 & 0.71 & 2.12 & 1.55 & 2.75 & 0.95 \\
5731 & -4.51 & 0.74 & 2.11 & 1.63 & $(2.5)$ & 0.96 \\
5775 & -4.46 & 0.79 & 2.18 & 1.50 & $(2.0)$ & 1.00 \\
5808 & -4.42 & 0.83 & 2.19 & 1.55 & $(1.5)$ & 1.05 \\
\hline
\end{tabular}

For our solar example, we set the surface gravity to $\log g=4.44 \mathrm{~cm} \mathrm{~s}^{-2}$ and used VALD to fix $\log g f$ and the relative values of $\Gamma_{6}$. Initially, we let $\Delta \Gamma_{6}$ vary with our other free parameters after which we fixed $\Delta \Gamma_{6}$ to particular values of interest. We then simultaneously varied the five remaining parameters: $T_{\text {eff }}, \log (\mathrm{Fe} / \mathrm{H}), V_{\text {micro }}, V_{\text {macro }}$, and $V \sin i$. Although the actual solar $T_{\text {eff }}$ is well determined, we let this parameter vary in our models in order to empirically determine the theoretical Kurucz model that best reproduces the observed spectrum. Rotation period as a function of solar latitude is also known, but the best choice for $V \sin i$ in a uniformly rotating model is unclear, so we solve for this parameter as well.

Table 2 gives solar parameters that we derived using SME. Iron abundances in column two are given as logarithmic ratios with respect to the total number of atoms. Parentheses around entries in column six indicate that $\Delta \Gamma_{6}$ was set to the tabulated value. The last column gives RMS residuals for points deeper than $1 \%$ of the line depth. Formal uncertainties are all much less than the tabulated precision, but systematic errors and correlated uncertainties are certainly larger.

Model convergence for the $\Delta \Gamma_{6}=2.5$ case required 68 spectrum synthesis operations, including calculation of eight separate partial derivatives with respect to five free parameters. Reduced $\chi^{2}$ improved from 5800 to 740 , changing by only 0.05 in the final iteration. The large value of $\chi^{2}$ implies that no combination of model parameters adequately reproduces a high quality solar spectrum. The line RMS of $0.96 \%$ is partially due to errors in $\log g f$, but the line shapes are also incorrect. In particular, observed line profiles are asymmetric (probably due to granulation), whereas our static, one-component models produce symmetric model profiles. The continuum RMS of $0.14 \%$ is well above the noise, due to line asymmetries and unidentified weak blends.

Table 3. Astrophysical $\log g f$ values determined by SME with associated atomic data and line-by-line fit quality

\begin{tabular}{|c|c|c|c|c|c|}
\hline $\begin{array}{c}\lambda \\
(\AA)\end{array}$ & $\begin{array}{c}\chi \mathrm{ex} \\
(\mathrm{eV})\end{array}$ & $\begin{array}{c}\text { Lab } \\
\log g f\end{array}$ & $\begin{array}{l}\text { Solar } \\
\log g f\end{array}$ & Transition & $\begin{array}{l}\text { Line } \\
\text { RMS }\end{array}$ \\
\hline 4389.245 & 0.052 & -4.583 & -4.562 & $a^{5} D_{3}-z^{7} F_{2}^{\circ}$ & 1.26 \\
\hline 4445.471 & 0.087 & -5.441 & -5.446 & $\mathrm{a}^{5} \mathrm{D}_{2}-\mathrm{z}^{7} \mathrm{~F}_{2}^{\circ}$ & 1.07 \\
\hline 5247.049 & 0.087 & -4.946 & -4.936 & $a^{5} D_{2}-z^{7} D_{3}^{\circ}$ & 0.76 \\
\hline 5250.208 & 0.121 & -4.938 & -4.886 & $a{ }^{5} D_{0}-z^{7} D_{1}^{\circ}$ & 0.76 \\
\hline 5701.545 & 2.559 & -2.216 & -2.149 & $\mathrm{~b}^{3} \mathrm{~F}_{2}{ }_{4}-\mathrm{y}{ }^{3} \mathrm{D}_{3}^{\circ}$ & 0.54 \\
\hline 5956.692 & 0.859 & -4.605 & -4.589 & $\mathrm{a}^{5} \mathrm{~F}_{5}-\mathrm{z}{ }^{7} \mathrm{P}_{4}^{\circ}$ & 0.99 \\
\hline 6082.708 & 2.223 & -3.573 & -3.614 & $a{ }^{5} \mathrm{P}_{1}-\mathrm{z}^{3} \mathrm{P}_{1}^{\circ}$ & 0.48 \\
\hline 6136.993 & 2.198 & -2.950 & -2.995 & $\mathrm{a}{ }^{5} \mathrm{P}_{2}-\mathrm{y}{ }^{5} \mathrm{D}_{1}^{\mathrm{o}}$ & 0.79 \\
\hline 6151.617 & 2.176 & -3.299 & -3.333 & $a{ }^{5} \mathrm{P}_{3}-\mathrm{y}{ }^{5} \mathrm{D}_{2}^{\circ}$ & 0.50 \\
\hline 6173.341 & 2.223 & -2.880 & -2.898 & $a^{5} \mathrm{P}_{1}-\mathrm{y}^{5} \mathrm{D}_{0}^{0}$ & 0.63 \\
\hline 6200.314 & 2.609 & -2.437 & -2.393 & $\mathrm{~b}{ }^{3} \mathrm{~F} 2_{2}-\mathrm{y}^{3} \mathrm{~F}_{3}^{\circ}$ & 0.77 \\
\hline 6219.279 & 2.198 & -2.433 & -2.492 & $\mathrm{a}{ }^{5} \mathrm{P}_{2}-\mathrm{y}{ }^{5} \mathrm{D}_{2}^{0}$ & 0.61 \\
\hline 6265.131 & 2.176 & -2.550 & -2.583 & $\mathrm{a}^{5} \mathrm{P}_{3}-\mathrm{y}{ }^{5} \mathrm{D}_{3}^{\circ}$ & 0.46 \\
\hline 6280.616 & 0.859 & -4.387 & -4.402 & $a^{5} F_{5}-z^{7} F_{5}^{0}$ & 1.08 \\
\hline 6297.792 & 2.223 & -2.740 & -2.774 & $a{ }^{5} \mathrm{P}_{1}-\mathrm{y}{ }^{5} \mathrm{D}_{2}^{\circ}$ & 0.65 \\
\hline 6322.690 & 2.588 & -2.426 & -2.384 & $\mathrm{~b}^{3} \mathrm{~F} 2_{3}-\mathrm{y}^{3} \mathrm{~F}_{4}^{\circ}$ & 0.69 \\
\hline 6481.869 & 2.279 & -2.984 & -2.962 & $\mathrm{a}{ }^{3} \mathrm{P} 2_{2}-\mathrm{y}{ }^{5} \mathrm{D}_{2}^{\circ}$ & 0.60 \\
\hline 6498.937 & 0.958 & -4.699 & -4.677 & $\mathrm{a}{ }^{5} \mathrm{~F}_{3}-\mathrm{z}^{7} \mathrm{~F}_{3}^{\circ}$ & 0.71 \\
\hline 6574.225 & 0.990 & -5.004 & -5.030 & $a^{5} F_{2}-z^{7} F_{2}^{\circ}$ & 0.30 \\
\hline 6593.871 & 2.433 & -2.422 & -2.396 & $\mathrm{a}^{3} \mathrm{H}_{5}-\mathrm{z}^{5} \mathrm{G}_{5}^{\circ}$ & 0.94 \\
\hline 6609.110 & 2.559 & -2.692 & -2.664 & $\mathrm{~b}{ }^{3} \mathrm{~F} 2_{4}-\mathrm{z}^{3} \mathrm{G}_{4}^{\circ}$ & 0.60 \\
\hline 6625.021 & 1.011 & -5.336 & -5.381 & $\mathrm{a}^{5} \mathrm{~F}_{1}-\mathrm{z}^{7} \mathrm{~F}_{1}^{\circ}$ & 0.21 \\
\hline 6750.150 & 2.424 & -2.621 & -2.594 & $\mathrm{a}{ }^{3} \mathrm{P} 2_{1}-\mathrm{z}{ }^{3} \mathrm{P}_{1}^{\circ}$ & 0.55 \\
\hline 6945.203 & 2.424 & -2.482 & -2.480 & $\mathrm{a}{ }^{3} \mathrm{P} 2_{1}-\mathrm{z}{ }^{3} \mathrm{P}_{2}^{\circ}$ & 0.47 \\
\hline 6978.850 & 2.484 & -2.500 & -2.474 & $\mathrm{a}{ }^{3} \mathrm{P} 2_{0}-\mathrm{z}^{3} \mathrm{P}_{1}^{\circ}$ & 0.45 \\
\hline 7723.205 & 2.279 & -3.617 & -3.521 & $\mathrm{a}{ }^{3} \mathrm{P} 2_{2}-\mathrm{z}{ }^{3} \mathrm{D}_{3}^{\circ}$ & 0.50 \\
\hline
\end{tabular}

\subsection{Comparison with expected values}

Before comparing individual parameters in Table 2 with expected values from the literature, we should emphasize again that parameters have correlated uncertainties and are subject to systematic errors. Each parameter has a unique effect on line shape, but no combination of parameters exactly matches the observed profiles. While attempting to match the observed profiles, parameters deviate from their true values by an unknown amount to partially compensate for deficiencies in the model. Such errors are difficult to quantify.

One way to explore possible systematic errors is to try a range of reasonable values for one parameter and solve for the remaining free parameters. The results of such an experiment are shown in Table 2 , where we set $\Delta \Gamma_{6}$ to 2.5 , 2.0 , and 1.5 , covering the range of values deemed acceptable by Holweger et al. (1991). The remaining five parameters were all varied to achieve the optimum fit. The fit 
with $\Delta \Gamma_{6}=1.5$ is significantly worse than the others, but the first three fits are all plausible, including a fit with $\Delta \Gamma_{6}=2.75$. The range of parameter values for these first three models gives one estimate of potential systematic errors.

For the purposes of discussion below, we will focus on the solar model with $\Delta \Gamma_{6}=2.5$. SME marginally favors $\Delta \Gamma_{6}=2.75$, but the difference in $\chi^{2}$ is negligible. The spectral lines in our study do not have strong damping wings, so they provide only moderate constraints on $\Delta \Gamma_{6}$. By the same token, our results are not very sensitive to the value we choose. Values of $\Delta \Gamma_{6}$ in the literature range from 1.5 to 2.5 (Holweger et al. 1991), motivating us to adopt a slightly lower than optimal value. We note in passing that the best value of $\Delta \Gamma_{6}$ apparently varies from element to element. No correction was needed in a similar SME analysis of a group of Ca I lines (Piskunov 1996).

Our favored model has $T_{\text {eff }}$ about $35 \mathrm{~K}$ below the Sun's actual $T_{\text {eff }}$ of $5770 \mathrm{~K}$. Such a discrepancy is possible, for example, if the $T_{\text {eff }}$ scale for the grid of model atmospheres is unrealistic. Spectra generated from semi-empirical solar models (HM, for example) usually match the observed solar spectrum better than synthetic spectra based on ab initio models. Theoretical models are hampered by uncertainties in atomic data and energy transport theory. On the other hand, very few stars have adequate data to constrain a semi-empirical model, so we are usually forced to consider theoretical models. Fortunately, theoretical models have improved significantly of late, due in large part to the extensive efforts of Bob Kurucz. Figure 1 compares the HM and Kurucz solar models with our best fitting semi-empirical model (which is interpolated from a grid of theoretical Kurucz models). Deep in the star, the SME model matches the empirical HM model, dropping to somewhat lower values at the surface.

Regarding the solar iron abundance, we find a value of $\log (\mathrm{Fe} / \mathrm{H})=-4.51$, which agrees well with studies of Fe I (Holweger et al. 1991), Fe II (Holweger et al. 1990), and the meteoric $\mathrm{Fe} / \mathrm{Si}$ ratio (Anders \& Grevesse 1989). Our formal errors are less than 0.01 , but there certainly exist larger systematic errors, which we will not attempt to characterize here. Although Blackwell et al. (1984) found a significantly higher value of $7.67 \pm 0.03$, BLS were able to reproduce either the "high" or the "low" value, depending on the model atmosphere they used in their analysis. In certain respects, our analysis is similar to that of BLS, including the set of spectral lines and their $\log g f$ values. One critical difference is that we match the line profiles, rather than equivalent widths. This is relevant since HKB have discussed at length the difficulties that may arise in determining equivalent widths for use in a traditional abundance analysis. Our $V_{\text {micro }}$ of $0.75 \mathrm{~km} \mathrm{~s}^{-1}$ is slightly lower than the $0.85 \mathrm{~km} \mathrm{~s}^{-1}$ of BLS, and significantly lower than the $1.0 \mathrm{~km} \mathrm{~s}^{-1}$ adopted by Holweger et al. (1991).

\subsection{Solar oscillator strengths}

SME can also be used to determine astrophysical values for atomic line parameters. As a demonstration, we again used the NSO solar atlas as a constraint, fixing the model parameters which couple to $\log g f$, i.e. $T_{\text {eff }}$, $\log g, \log (\mathrm{Fe} / \mathrm{H}), V_{\text {micro }}$, and $\Delta \Gamma_{6}$. These model parameters were set to the values determined in the SME run with $\Delta \Gamma_{6}=2.5$ described in the previous section. Using SME with this model, we solved for more precise astrophysical $\log g f$ values, which remain tied to the absolute scale of the BLS $\log g f$ measurements used to construct the model. We also allowed $V_{\text {macro }}$ and $V \sin i$ to vary, as these parameters affect only line shape, not line strength. As expected, $V_{\text {macro }}$ and $V \sin i$ did not change significantly. $\chi^{2}$ dropped to 407 , and the line and continuum RMS values were $0.70 \%$ and $0.13 \%$. Tuning the $\log g f$ values yields a substantially better fit, but significant discrepancies remain. Asymmetries in the line profiles suggest that the model used here should be enhanced to include the effects of correlated temperature and velocity distributions in the photosphere.

Figure 2 shows our fit to the observations for all the lines in our list, while Fig. 3 demonstrates how adjusting the oscillator strengths affects the fit. Column 4 of Table 3 gives the astrophysical $\log g f$ values determined with SME, which are to be compared with the laboratory values in Col. 3. Figure 4 shows the ratio of solar to laboratory oscillator strengths minus one. The discrepancies are consistent with the $9 \%$ uncertainty quoted by Bard \& Kock (1994), but not with the $2 \%$ uncertainties claimed by BLS. For low excitation lines, there is no obvious pattern to the residuals, but as line excitation increases above $2 \mathrm{eV}$, solar $\log g f$ values generally increase, relative to laboratory $\log g f$. This suggests a systematic error either in the model atmosphere or in the laboratory data. In either case, using astrophysical $\log g f$ improves agreement with observed spectra at the cost of a possible increase in systematic errors, especially when extending results to significantly different spectral types.

\section{Conclusions and future work}

We have developed a new tool for extracting information from stellar spectra by fitting them with synthetic spectra. SME provides an LTE spectral synthesis code together with an intuitive graphics interface to specify fitting tasks and analyze results. A variety of tests based on the NSO Solar Atlas demonstrate that SME is capable of determining both global stellar parameters and astrophysical data for individual atomic transitions.

In the near future we intend to use SME to identify which spectral lines are the best gravity and temperature diagnostics, particularly for late-type stars. These diagnostics will be valuable, for example, in establishing the relation between the temperature scales based on 


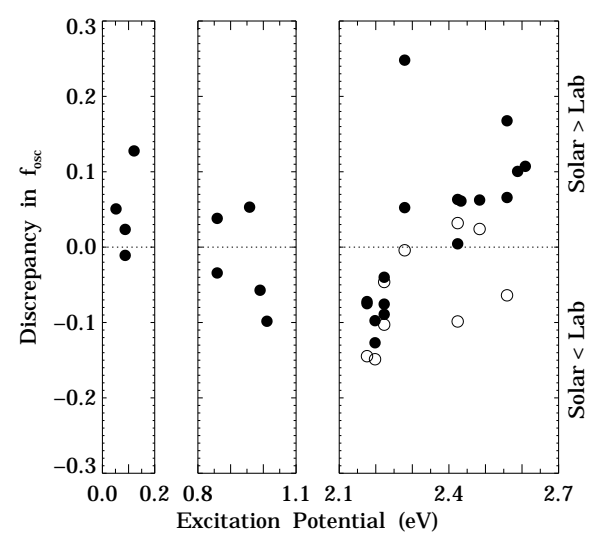

Fig. 4. Discrepancy between laboratory and astrophysical oscillator strengths, expressed as the ratio of solar to laboratory $f_{\text {osc }}$ minus one. Positions of the filled circles were calculated using $\log g f$ values from BLS, whereas the unfilled circles used $\log g f$ values from Bard \& Kock (1994)

photometry and on model atmospheres. Another immediate application of SME is the verification and enhancement of atomic line data. Even in our solar example, we encountered observed lines that could not be associated with any line in the VALD data base, which is the largest collection of atomic lines. On the other hand, synthetic spectra generated using VALD atomic data also contained a few lines that were not present in the solar spectrum. Using SME to fit high quality spectra of several objects with different temperatures and gravities will provide a very thorough test for the quality and completeness of current line lists. For lines without laboratory data, we will be able to determine astrophysical $\log g f$ values, which can be used until accurate laboratory measurements are available.

We are also considering several enhancements of SME. Among the possible improvements, we recognize these as the most urgent:

- multicomponent models with granular flows,

- effects of a magnetic field,

- including NLTE effects,

- including molecular line opacity.

Regular use of tools like SME requires intensive access to a comprehensive atomic database. For that reason, we intend to integrate SME into VALD, making it a standard VALD tool that can be used to interpret a wide range of spectroscopic observations. SME itself may be obtained by writing directly to the authors.
This work was supported in part by NASA grant NAGS-23007 to the University of Colorado.

\section{References}

Bard A., Kock M., 1994, A\&A 282, 1014

Blackwell D.E., Booth, A.J., Petford, A.D., 1984, A\&A 132, 236

Blackwell D.E., Lynas-Gray A.E., Smith G., 1995, A\&A 296, 217 (BLS)

Cowley C., 1971, Observatory 91, 139

Cowley C.R., 1996, in Proceedings of the 5th International workshop on model atmospheres and spectral synthesis, Vienna, 1995 (in press)

Dimitrijević M.S., Sahal-Bréchot S., 1990, A\&AS 82, 519

Gray D.F., 1992, The Observation and Analysis of Stellar Photospheres. Cambridge University Press, Cambridge

Griem H.R., 1968, Phys. Rev. 165, 258

Hill G., Gulliver A.F., Adelman S.J., 1996, in Proceedings of the 5th International workshop on model atmospheres and spectral synthesis, Vienna, 1995 (in press)

Holweger H., Bard A., Kock A., Kock M., 1991, A\&A 249, 545

Holweger H., Kock M., Bard A., 1995, A\&A 296, 233 (HKB)

Humlíček J., 1982, J. Quant. Spectrosc. Rad. Transf. 27, 437

Kurucz R.L., 1979, SAO Special Report No. 309

Kurucz R.L., Furenlid I., Brault J., Testerman L., 1984, National Solar Observatory Atlas No. 1: Solar Flux Atlas from 296 to $1300 \mathrm{~nm}$. National Solar Observatory, Sunspot

Kurucz R.L., 1993, CD-ROM No. 18: ATLAS9 Stellar Atmosphere Programs and $2 \mathrm{~km} / \mathrm{s}$ Grid

Marquardt D.W., 1963, J. Soc. Ind. Appl. Math. 11, 431

Piskunov N.E., 1992, in: Proceedings of the International Meeting "Stellar Magnetism", Special Astrophys. Observatory, Nizhnij Arkhiz, North Caucasus, September 1991. In: Glagolevskij Yu.V., Romanuk I.I. (eds.), Nauka, St. Petersburg, p. 92

Piskunov N.E., 1996, A\&A (in preparation)

Piskunov N.E., Kupka F., Ryabchikova T.A., Weiss W.W, Jeffery C.S., 1995, A\&AS 112, 525

Press W.H., Flannery B.P., Teukolsky S.A., Vetterling W.T., 1986, Numerical Recipes. Cambridge University Press, Cambridge, p. 523

Unsöld A., 1955, Physik der Sternatmosphären. SpringerVerlag, Berlin

Valenti J.A., Anderson J., 1995, PASP (in preparation)

Vidal C.R., Cooper J., Smith E.W., 1971, J. Quant. Spectrosc. Rad. Transf. 11, 263

Vincent A., Piskunov N.E., Tuominen I., 1993, A\&A 278, 523 